\title{
miR-511-3p, embedded in the macrophage mannose receptor gene, contributes to intestinal inflammation
}

SE M Heinsbroek ${ }^{1}$, ML Squadrito $^{2}$, R Schilderink ${ }^{1}$, FW Hilbers ${ }^{1}$, C Verseijden $^{1}$, M Hofmann $^{1}$, A Helmke $^{1}$,

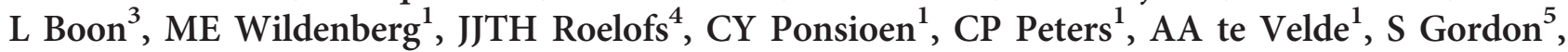
M De Palma ${ }^{2}$ and WJ de Jonge ${ }^{1}$

MiR-511-3p is embedded in intron 5 of the CD206/MRC1 gene Mrc1, expressed by macrophage and dendritic cell populations. CD206 and miR-511-3p expression are co-regulated, and their contribution to intestinal inflammation is unclear. We investigated their roles in intestinal inflammation in both mouse and human systems. Colons of CD206deficient mice displayed normal numbers of monocytes, macrophage, and dendritic cells. In experimental colitis, CD206-deficient mice had attenuated inflammation compared with wild-type (WT) mice. However, neither a CD206 antagonist nor a blocking antibody reproduced this phenotype, suggesting that CD206 was not involved in this response. Macrophages isolated from CD206-deficient mice had reduced levels of miR-511-3p and TIr4 compared with WT, which was associated with reduced pro-inflammatory cytokine production upon lipopolysaccharides (LPS) and fecal supernatant stimulation. Macrophages overexpressing miR-511-3p showed $50 \%$ increase of TIr4 mRNA, whereas knockdown of miR-511-3p reduced TIr4 mRNA levels by $60 \%$, compared with scrambled microRNA (miRNA)-transduced cells. Response to anti-tumor necrosis factor (TNF) treatment has been associated with elevated macrophage CD206 expression in the mucosa. However, in colon biopsies no statistically significant change in miR-511-3p was detected. Taken together, our data show that miR-511-3p controls macrophage-mediated microbial responses and is involved in the regulation of intestinal inflammation.

\section{INTRODUCTION}

The macrophage mannose receptor CD206 is a pattern recognition receptor expressed by macrophage and dendritic cell (DC) populations. ${ }^{1,2} \mathrm{CD} 206$ recognizes a broad range of microbes including fungi, protozoa, bacteria, parasites, and viruses. ${ }^{1}$ Although it is unclear whether this receptor is capable of signal transduction, it has been shown to mediate endocytosis and influence antigen processing upon ligand recognition. ${ }^{3,4}$ Owing to its ability to recognize a large diversity of microbes and modulate immune responses, we hypothesized that CD206 may be an important factor in intestinal inflammation.
An additional interesting feature of CD206 is that it harbors a microRNA (miRNA), miR-511-3p, within intron 5 of the gene (Mrc1). MiR-511-3p expression has profound functional effects on macrophages. ${ }^{5}$ MiRNAs are short (18-24 nucleotides in length), endogenous, noncoding single-stranded RNAs that regulate gene expression by controlling the stability and translation of protein-coding mRNAs. MiR-511-3p is the functional miRNA strand in the precursor (pre)-miR-511. MiR-511-3p levels correlate with CD206 expression and control the activation of tumor-associated macrophages. ${ }^{5}$ Inhibiting the pre-miR-511 has been shown to decrease Toll-like receptor-4 (TLR4) levels in human monocytes. ${ }^{6}$

${ }^{1}$ Academic Medical Center, Tytgat Institute for Liver and Intestinal Research, University of Amsterdam, AMC, Amsterdam, The Netherlands. ${ }^{2}$ The Swiss Institute for Experimental Cancer Research (ISREC), École Polytechnique Fédérale de Lausanne, Lausanne, Switzerland. ${ }^{3}$ EPIRUS Biopharmaceuticals Netherlands BV, Utrecht, The Netherlands. ${ }^{4}$ Department of Pathology, Academic Medical Center, University of Amsterdam, AMC, Amsterdam, The Netherlands and ${ }^{5}$ Sir William Dunn School of Pathology, University of Oxford, Oxford, UK. Correspondence: SEM Heinsbroek (s.e.heinsbroek@amc.uva.nl) 
Aberrant TLR signaling contributes to intestinal inflammation, ${ }^{7}$ indicating that miR-511-3p may interact with TLR4 gene expression to control macrophage activation and differentiation.

In the current study, we show that $\mathrm{Mrc1}^{-1-}$ mice develop less severe dextran sulfate sodium (DSS)-induced colitis in both acute and chronic colitis settings. We demonstrate that the CD206-binding site seems not to be directly involved in this response, as blocking this site with a CD206-specific antibody or chemical antagonist did not affect the course of colitis. Instead, we show that CD206-deficient macrophages have reduced levels of miR-511-3p (confirming recent studies) ${ }^{5,8}$ and Tlr4, and display a reduced inflammatory response to lipopolysaccharides (LPS) stimulation. When miR-511-3p expression was experimentally reduced in bone marrow-derived macrophages (BMDMs), these cells had an impaired cytokine response to LPS. These data suggest that miR-511-3p is an important determinant of macrophage responses to LPS.

\section{RESULTS}

\section{CD206-deficient mice are protected against DSS-induced} colitis

To address the role of CD206 in intestinal immune homeostasis, we first determined the presence of CD206-expressing cells in the colon lamina propria of wild-type (WT) mice. Around 2\% CD45-expressing lamina propria cells express CD206 in healthy conditions. In the course of DSS-induced colon inflammation, the number of CD206-expressing cells increased significantly (Figure 1a). In both healthy (Figure 1b) and colitic (Figure 1c) mice, CD206 and CD11b immunolabeling showed the clear presence of CD206positive cells that co-stained with CD11b (Figure 1a and b). We next aimed to test CD206 involvement in colitis. Colitis in WT and $\mathrm{MrCl}^{-1-}$ mice was induced using DSS in the drinking water in an acute (7-day DSS) and chronic (30 days after start of DSS) colitis setting. Both WT and $\mathrm{Mrcl}^{-1-}$ mice started losing weight on day 5 ; weight recovery started on day 10 , and on day 17 both groups of mice had regained $100 \%$ of their initial body weight (Figure $\mathbf{2 b}$ ). The course of colitis was determined at 3,7, and 30 days after DSS administration by determining colon weight and inflammation score. On day 7 , we observed a significantly lower colon weight and lower inflammation score in $\mathrm{Mrcl}^{-1-}$ mice compared with WT mice (Figure 2c and $\mathbf{d}$ ), indicating that $\mathrm{Mrcl}^{-1-}$ mice are protected from colitis. Histological inflammation scoring of the intestine showed that $\mathrm{MrCl}^{-1-}$ mice had a reduced influx of inflammatory cells, less crypt loss, and less epithelial erosion on day 7 (Figure 2d and e). Signs of inflammation were still noticeable on day 30 in WT mice, whereas $M r c 1^{-1-}$ mice had recovered from DSS colitis as indicated by significantly lower colon weight and lower inflammation scores at this time point of analysis.

We next assessed more specific parameters of inflammation on day 7 of DSS colitis (Figure 2f). At this time point, WT mice often had soft and sometimes even bloody stools with a score of 2 and higher, whereas the faeces score of $\mathrm{Mrcl}^{-1-}$ mice was close to untreated (non-colitis) mice, which have a score of 0
(Figure 2f). Pro-inflammatory cytokines were measured in colon lysates, showing that $\mathrm{Mrcl}^{-1-}$ mice had significantly attenuated levels of CCL2 and IL-6 compared with WT (Figure 2f). Consistent with reduced colonic inflammation, colon infiltrates of $\mathrm{CD} 45^{+} \mathrm{CD} 11 \mathrm{~b}^{+} \mathrm{CD} 11 \mathrm{c}^{+}, \mathrm{CD} 45^{+}$ $\mathrm{CD} 11 \mathrm{~b}^{+} \mathrm{CD} 11 \mathrm{c}^{-}$immune cells, were significantly reduced in $\mathrm{MrCl}^{-1-}$ mice compared with WT mice (Supplementary Figure 1 online). Together, these data indicate that $\mathrm{Mrcl}^{-1-}$ mice, in comparison with WT mice, have reduced inflammatory cell infiltrates and histological signs of colitis, and thus are protected against colonic inflammation.

Blocking CD206 does not affect the course of inflammation The carbohydrate recognition domains of CD206 mediate microbial recognition. We therefore hypothesized that blocking these domains would alternatively reduce intestinal inflammation and replicate the phenotype observed in $\mathrm{Mrcl}^{-1-}$ mice. To this aim, we use both a blocking antibody and soluble Saccharomyces cerevisiae mannan to antagonize CD206 carbohydrate recognition. The antibody, 5D3, binds and blocks the carbohydrate recognition domains, which are involved in microbial recognition. ${ }^{9}$ Mice were given DSS in the drinking water from day 1 up to day 7. On days 3 and 10, the mice received an intraperitoneal injection of 1-mg 5D3 or isotype control (Figure 3a), a dose shown appropriate to neutralize CD206 protein systemically. ${ }^{9}$ Intestinal inflammation was evident from body weight loss, compared with mice without DSS in their drinking water (Figure 3b). No significant difference in body weight loss was found between mice treated with 5D3 or isotype control antibody. Similarly, we found no difference between isotype-treated and 5D3-treated WT mice in body weight, colon weight, and inflammation score in DSS colitis (Figure 3c). Next, we assessed the effect of blocking CD206 using soluble S. cerevisiae mannan. Mannan is widely used as an antagonist of CD206 to inhibit microbial recognition. ${ }^{10-13}$ One milligram soluble $S$. cerevisiae mannan was given to the mice daily for 14 days by oral gavage, followed by 7-day DSS treatment (Figure 3d). No significant difference in body weight loss was found between vehicle and mannan-fed mice (Figure 3e). We also did not detect any difference between vehicle and mannan-fed mice in colon weight and inflammation score (Figure 3f). Together, these data show that the carbohydrate recognition domain of CD206 is not involved in the pathogenesis of DSS-induced intestinal inflammation and that the lack of CD206 does not readily explain protection against colitis.

\section{CD206-deficient macrophages display a reduced pro-inflammatory cytokine response compared with WT macrophages upon LPS stimulation}

We next investigated cytokine production in response to microorganisms in CD206-deficient macrophages. To study the role of CD206 in this process, mouse WT or $\mathrm{MrCl}^{-/}$ BMDMs were polarized into M1 $\left(\mathrm{CD} 206^{\text {low }}\right)$ or CD206 ${ }^{\text {high }}$ expressing M2 macrophages by using either IFN $\gamma$ or IL-4, respectively. To simulate a microbial encounter, LPS was added to the cells. $\mathrm{Mrcl}^{-1-}$ macrophages showed more than 50\% 
a

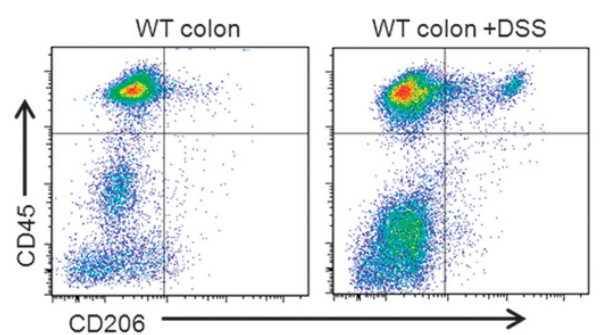

b
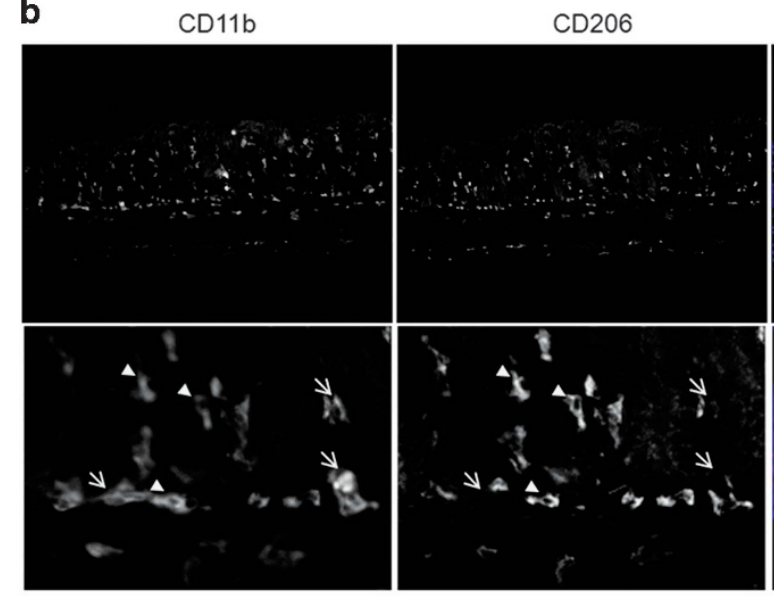

C
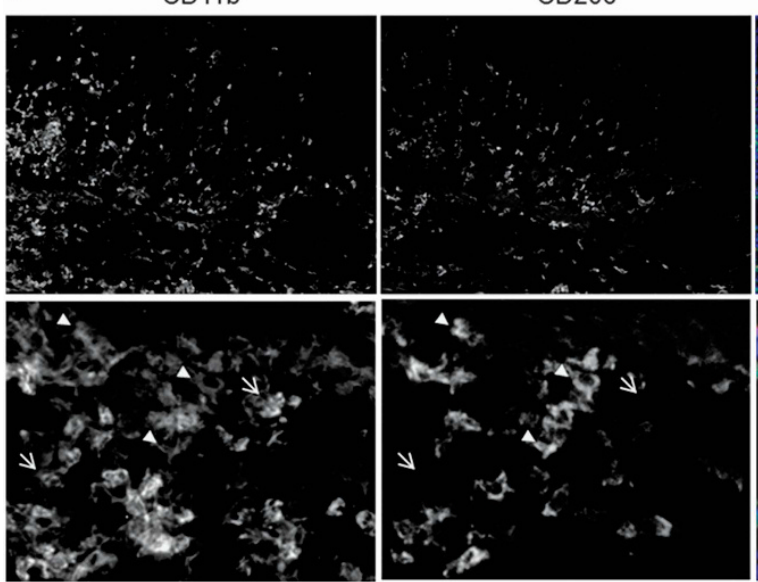

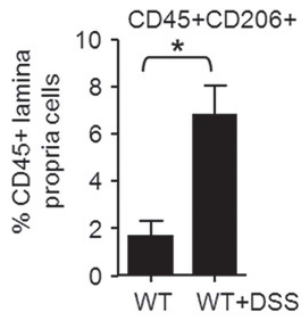

DAPI CD11b CD206

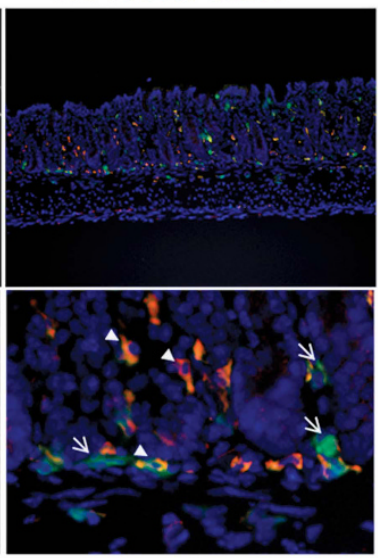

DAPI CD11b CD206
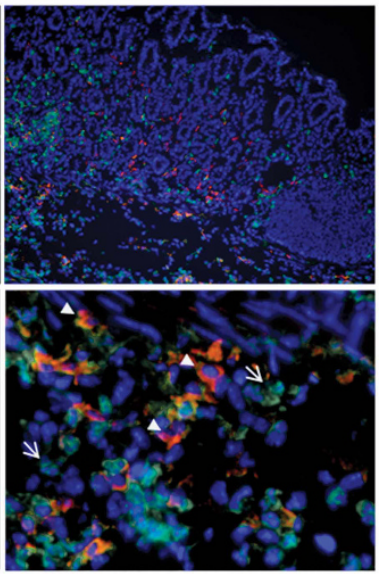

Figure 1 CD206 expression in healthy and inflamed mouse colon. (a) Flow cytometry analysis of the expression of CD206 in healthy mouse colon and in inflamed colon after 7 days of dextran sulfate sodium (DSS)-induced colitis. $N=3$ for each group. A $t$-test was used for statistical analysis. ${ }^{\star} P<0.05$. (b) Fluorescent immunohistochemistry showing 4,6-diamidino-2-phenylindole (DAPI), CD11b, and CD206 in the healthy intestine showing that CD206expressing cells also express CD11b (arrowheads). Not all cells that express CD11b express CD206 (arrows). (c) Representative image of inflamed colon after 7 days of DSS-induced colitis showing an increase in CD11b- and CD206-expressing cells in the lamina propria.

reduced levels of Il6 and Tnfa mRNA compared with WT, irrespective of their M1/M2 differentiation (Figure 4a). Levels of Tnfa were similar between M1 and M2 macrophages, and Il6 levels were lower for M2 macrophages. Ccl2 mRNA levels were significantly higher in M2 macrophages but no differences were found in $\mathrm{Ccl} 2$ levels between WT and $\mathrm{Mrcl}^{-1-}$ macrophages. In contrast, $\mathrm{Ccl} 2$ levels were reduced in colon tissues from $\mathrm{MrCl}^{-1-}$ mice, compared with WT mice, in DSS-induced colitis, which likely represents reduced colitis in the $\mathrm{Mrcl}^{-1-}$ mice. Interestingly, $\mathrm{Mrcl}^{-1-}$ macrophages also showed reduced levels of Tlr2 and Tlr4 mRNA, in parallel with the reduced LPS responses (Figure $\mathbf{4 b}$ and c). Notably, Tlr 2 levels were higher in M1 WT macrophages compared with $\mathrm{MrCl}^{-1-}$, and Tlr2 levels were less abundant on M2 macrophages of either WT or $\mathrm{MrCl}^{-1-}$ genotype (Figure 4c).

It has been shown that the mature miR-511-3p, located within the fifth intron of the mouse and human Mrc1/MRC1 genes, is the functional miRNA strand in the precursor (pre)miR-511. ${ }^{5,14,15}$ Inhibiting the pre-miR-511 has been shown to indirectly decrease Tlr4 levels in monocytes/macrophages. ${ }^{6}$ 
a

DSS colitis model

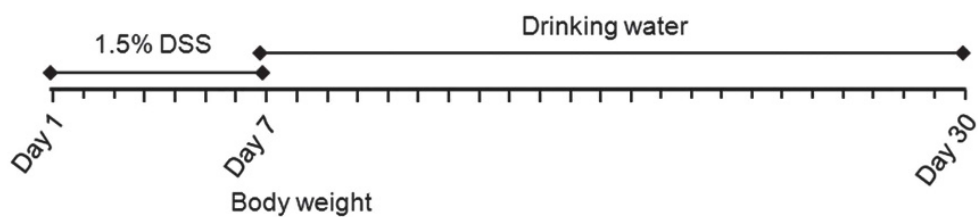

b

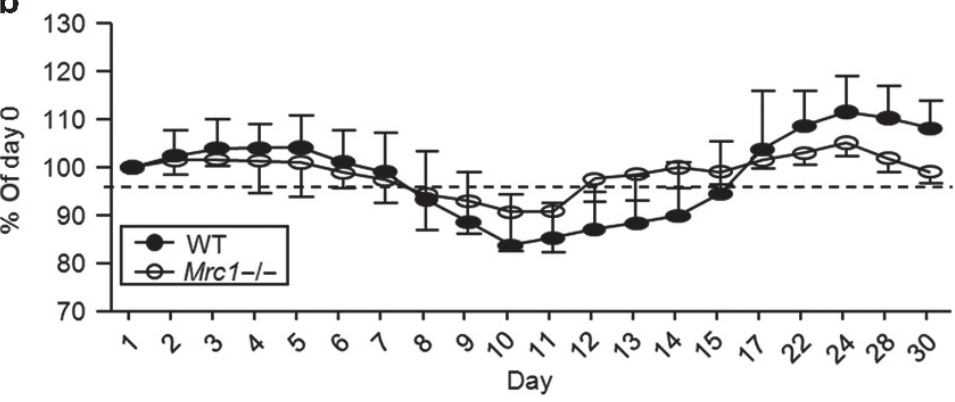

C

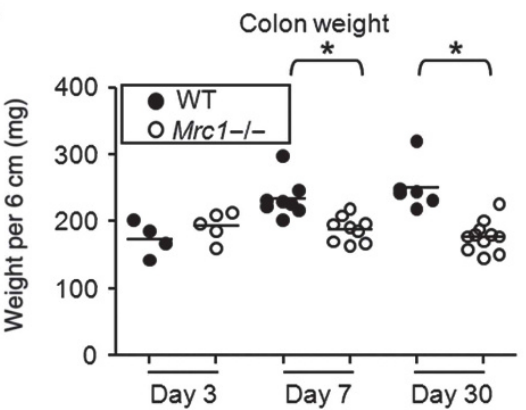

d Inflammation score

e

WT
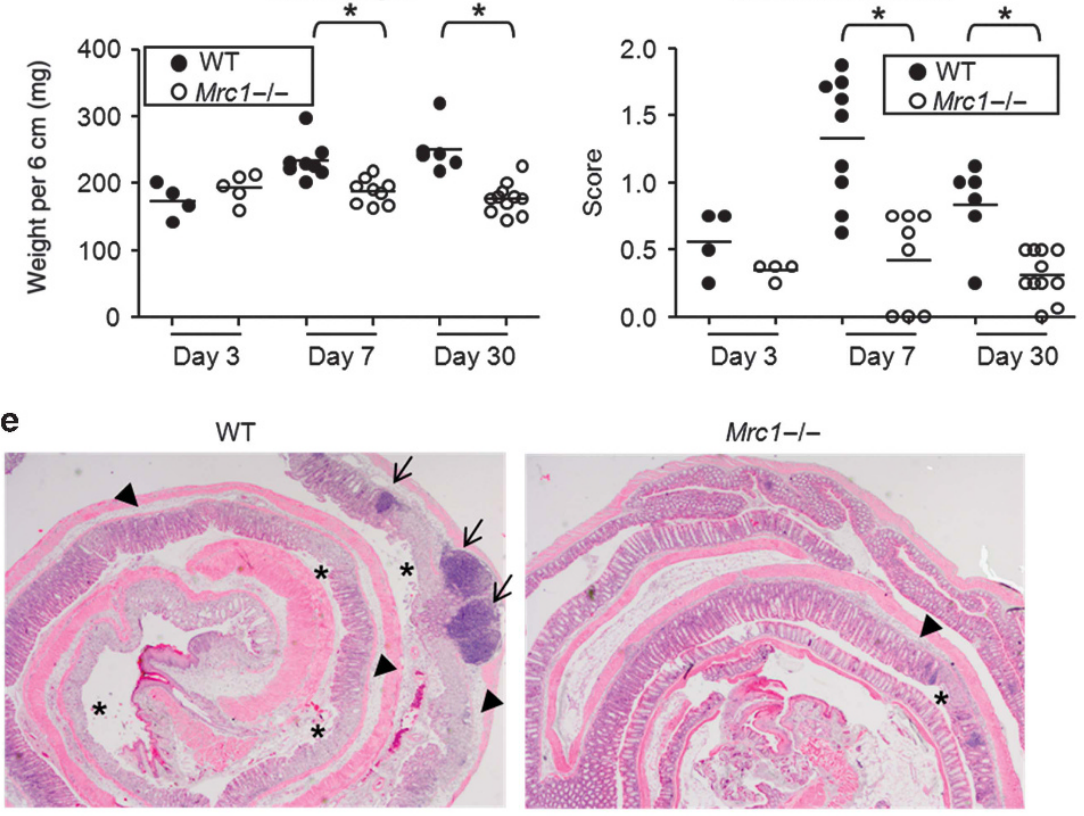

f
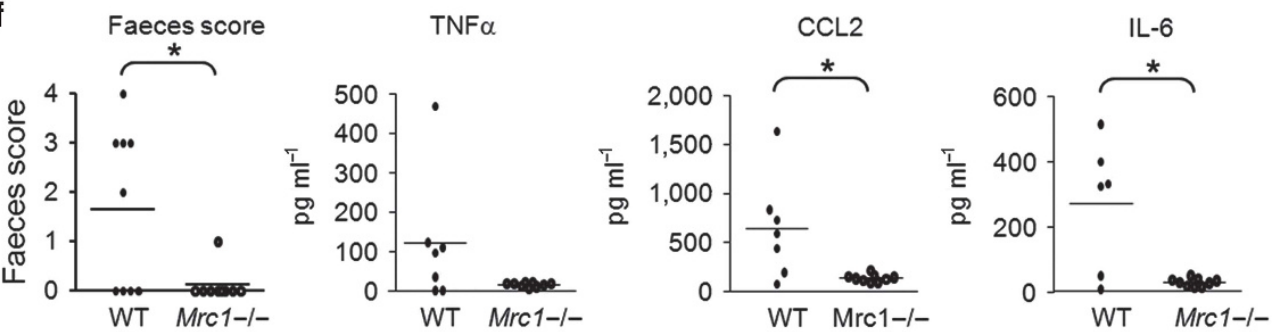

Figure 2 CD206-deficient mice develop less dextran sulfate sodium (DSS)-induced intestinal inflammation. (a) DSS colitis was induced by giving 1.5\% (w/v) DSS in drinking water. Mice were killed after 3,7 , and 30 days to determine colon weight and inflammatory scores. (b) Body weights were measured as indication of disease severity in this model; weights are shown as percentage of weight compared with those on day 1. (c) Colons were weighed as a measure of inflammation, shown as weight per 6 -cm colon. (d) Colon inflammation scores were determined by an experienced pathologist. Colons were graded from 0 to 4 points as indicated in Supplementary Table I. (e) Representative picture of WT and Mrc $1^{-1-}$ colon after 7 days of DSS-induced inflammation. In both groups, areas of cryptloss (asterisk) and edema were present (arrowheads). Gut-associated lymphoid tissue was found in areas of extensive inflammation (arrows). (f) Faeces scores and cytokine protein concentrations in colon lysates after 7 days with DSS. The sample means are indicated with a line. A $t$-test was used for statistical analysis. ${ }^{\star} P<0.05$. 


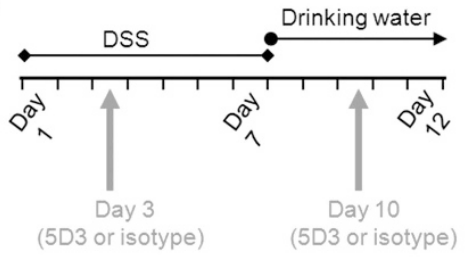

b

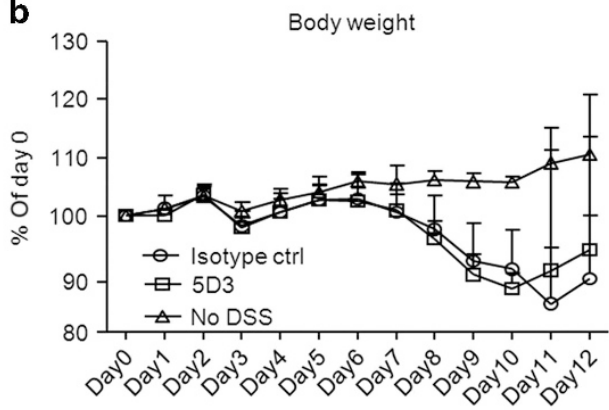

C

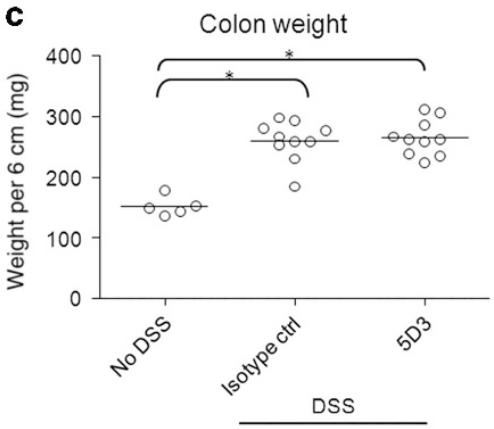

d

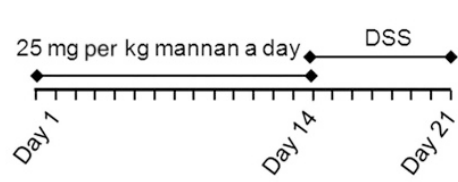

f

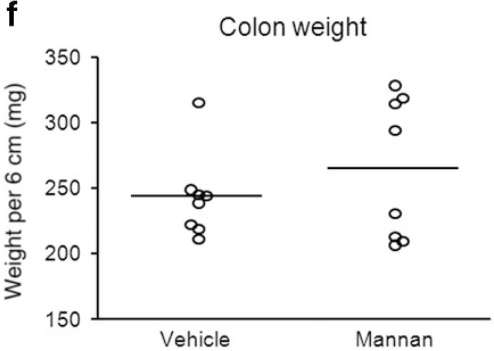

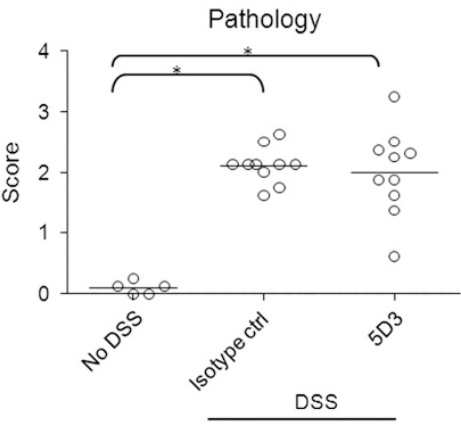

e
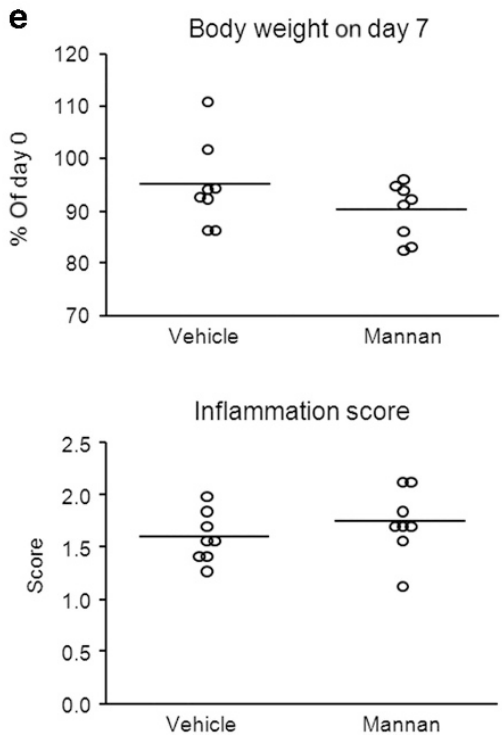

Figure 3 CD206 antagonists do not affect dextran sulfate sodium (DSS)-induced intestinal inflammation. (a) DSS colitis was induced by giving $1.5 \%$ $(\mathrm{w} / \mathrm{v})$ DSS in drinking water. On days 3 and 10, mice were injected intraperitoneally with $1 \mathrm{mg} 5 \mathrm{D} 3$ or isotype control antibody. After 12 days, colon weight and inflammatory scores were determined. $n=10$ for mice receiving DSS and $n=5$ for no DSS control. (b) Body weights were measured as an indication of disease severity in this model; weights are shown as percentage of weight compared with those on day 1. (c) Colons were weighed as a measure of inflammation, shown as weight per 6-cm colon and colon inflammation scores were determined by an experienced pathologist. Colons were graded from 0 to 4 points as indicated in Supplementary Table I. (d) Mice were given $1 \mathrm{mg}$ soluble Saccharomyces cerevisiae mannan a day for 14 days after which DSS colitis was induced by giving $1.5 \%$ (w/v) DSS in drinking water for 7 days, $n=8$ for each group. (e) Body weights were measured at the end of this experiment as an indication of disease severity; weights are shown as percentage of weight compared with those on day 1. (f) Colons were weighed as a measure of inflammation, shown as weight per 6 -cm colon, and colon inflammation scores were determined by an experienced pathologist. Colons were graded from 0 to 4 points as indicated in Supplementary Table I. The sample means are indicated with a line. A one-way analysis of variance (ANOVA) with Bonferroni multiple comparison test was used for statistical analysis. ${ }^{*} P<0.05$.

Therefore, we hypothesized that miR-511-3p, rather than CD206 itself, is responsible for the reduced inflammatory responses observed either upon LPS stimulation in $\mathrm{Mrcl}^{-1-}$ macrophages or in DSS-induced colitis in $\mathrm{Mrcl}^{-1-}$ mice. We found that $\mathrm{MrCl}^{-1-}$ macrophages have at least five times lower levels of miR-511-3p than WT macrophages, irrespective of M1 or M2 maturation (Figure $\mathbf{4 d}$ and $\mathbf{e}$ ). This confirms that the activity of the $\mathrm{Mrcl}$ gene positively co-regulates miR-511-3p expression and that genetic Mrc1 deletion abates miR-511-3p expression in our model. Whereas CD206 was strongly 

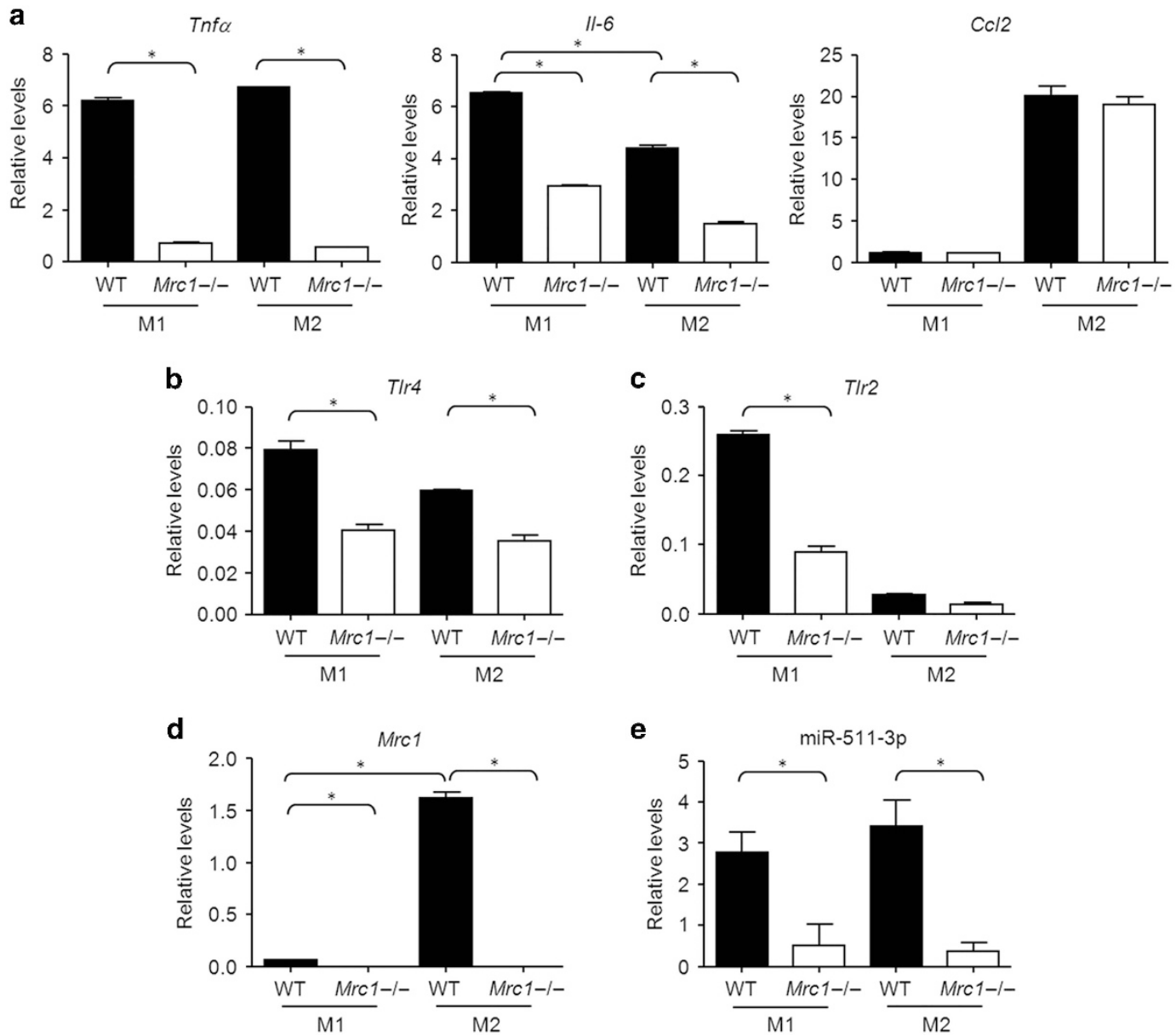

Figure 4 CD206-deficient macrophages produce less cytokine and contain reduced levels of TLR2, TLR4 and miR-511-3p. (a) Bone marrow-derived macrophages (BMDMs) from wild-type (WT; black bars) or $\mathrm{Mrc1}^{-1-}$ (white bars) mice were cultured for 2 days with interferon- $\gamma$ (IFN $\gamma$; M1) or interleukin (IL-4; M2) and subsequently stimulated for $4 \mathrm{~h}$ with LPS. RNA levels of Ccl2, Tnfo, and Il-6 were measured in cell lysates. (b) TIt4, (c) T/r2, (d) Mrc1, and (e) miR-511-3p levels were determined in WT (black bars) or Mrc1 ${ }^{-1}$ (white bars) BMDMs cultured for 2 days with interferon- $\gamma$ (IFN $\gamma$; M1) or IL-4 (M2). These data are representative for four independent experiments; error bars indicate the s.d. A one-way analysis of variance (ANOVA) with Bonferroni multiple comparison test was used for statistical analysis. ${ }^{\star} P<0.05$.

upregulated in M2-polarized cells (Figure 4d), miR-511-3p expression increased in both M1 and M2 macrophages compared with unstimulated cells (Figure 4e). Hence, in contrast to two earlier reports, ${ }^{5,16}$ the expression of miR-511-3p and CD206 does not tightly correlate in our M1/M2 differentiation systems, possibly explained by differences in miRNA vs. protein stability and turnover, or the cytokine-polarization protocol used.

\section{miR-511-3p is expressed in WT, but not in $\mathrm{MrC1}^{-1-}$ colonic macrophages}

We next examined the exact subpopulation of lamina propria mononuclear phagocytes that express CD206 and miR-511-3p. As CD206 expression is restricted to macrophage and DC populations, ${ }^{1,2}$ we focused on colonic $\mathrm{CD} 11 \mathrm{~b}^{+} \mathrm{Ly} 6 \mathrm{G}^{-} \mathrm{CD} 64^{-}$

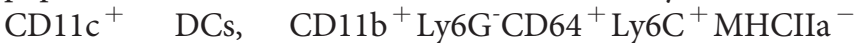

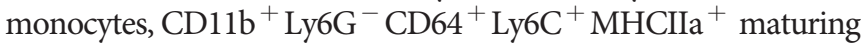

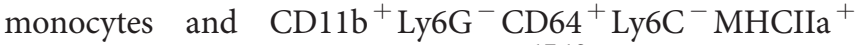
macrophages, as established earlier ${ }^{17,18}$ (Figure 5a). No differences in the relative distribution of DC, monocyte, maturing monocyte, and macrophages in the lamina propria were found between WT and $\mathrm{Mrcl}^{-1-}$ mice (Figure $5 \mathbf{b}-$ top graphs), indicating that $\mathrm{Mrcl}$ deficiency does not affect resident myeloid populations under control conditions. We found that mainly macrophages and, to a lesser extent, maturing monocytes expressed $M r c 1$, whereas monocytes and DCs express minimal levels of Mrc1. MiR-511-3p was only detected in macrophages (Figure 5b). $\mathrm{MrCl}^{-1-}$ macrophages are not only deficient for $M r c 1$ but also lack miR-511-3p (Figure 5b). Consistent with our BMDM experiments, expression of Trl4 was reduced in $\mathrm{Mrcl}-/$ - monocyte and macrophage subsets compared with WT, although this difference only reached significance in the $\mathrm{Mrcl}-/-$ monocyte fraction (Figure 5b).

miR-511-3p negatively regulates TLR4 expression levels To further investigate the role of miR-511-3p in the control of Tlr4 expression, we used immortalized BMDMs engineered to overexpress or knockdown miR-511-3p levels. ${ }^{8}$ miR-511-3p 
a
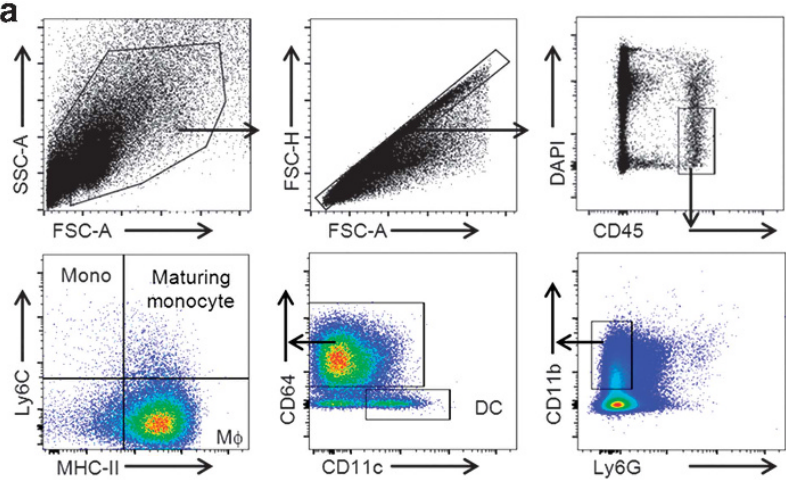

b

Cell numbers

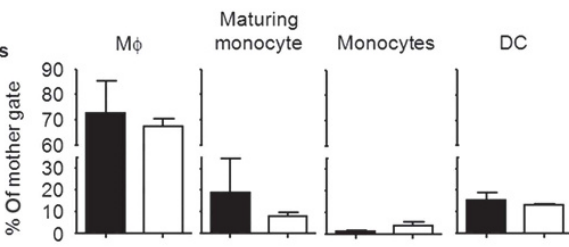

Mrc1

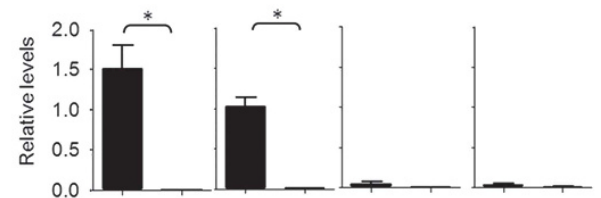

$\operatorname{miR}-511-3 p$

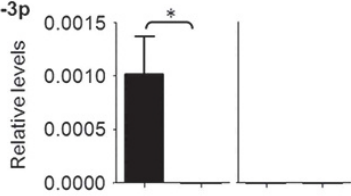

TIr4

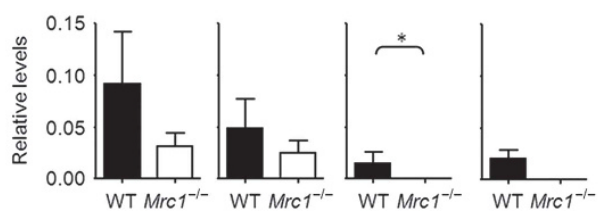

Figure 5 Mrc1 and miR-511-3p levels in colonic lamina propria cells. (a) Colonic CD11b ${ }^{+} \mathrm{CD} 64^{-} \mathrm{CD}_{11 \mathrm{c}}{ }^{+} \mathrm{DCs}, \mathrm{CD} 11 \mathrm{~b}{ }^{+} \mathrm{CD} 64^{+} \mathrm{y} 6 \mathrm{C}^{+}$ $\mathrm{MHCa}^{-}$monocytes, $\mathrm{CD} 11 \mathrm{~b}^{+} \mathrm{CD} 64^{+} \mathrm{Ly}_{6 \mathrm{C}}{ }^{+} \mathrm{MHCa}^{+}$maturing monocytes and $\mathrm{CD} 11 \mathrm{~b}^{+} \mathrm{CD} 64^{+} \mathrm{Ly}_{6 \mathrm{C}^{-}} \mathrm{MHCa}^{+}$macrophages were isolated using flow cytometry sorting. Debri was excluded using FSC-H and SSC-A gating, followed by excluding doublets by FSC-A and FSC-H gating. Live leukocytes were selected as $\mathrm{DAPI}^{-} \mathrm{CD} 45^{+}$. The resulting cells were then analyzed for CD11b, Ly6G, CD11c, CD64, MHCII, and Ly6C. (b) Percentage of colonic monocytes, maturing monocytes, dendritic cells (DCs), and macrophages in mouse colons and levels of Mrc1, miR-511-3p, and TIr4 in these populations. These data are representative for two independent experiments with 5-11 mice per group. Error bars indicate the s.d. A one-way analysis of variance (ANOVA) with Bonferroni multiple comparison test was used for statistical analysis. ${ }^{\star} P<0.05$.

knockdown was achieved using a 'sponge' LV overexpressing an antisense miRNA complementary to miR-511-3p, which prevents binding of miR-511-3p to its natural targets. ${ }^{8,19}$

We first assessed whether overexpression of miR-511-3p modulated functional characteristics attributed to M1 and M2 macrophages. We made use of the fact that M1- and M2polarized macrophages are functionally and metabolically characterized by distinct bioenergetics profiles. ${ }^{20}$ Glucose uptake and oxidative phosphorylation were, therefore, evaluated using an extracellular flux analyzer in mouse macrophages stably transduced with LVs (in order to overexpress scrambled or specific miR511-3p) and further polarized to M1 or M2. Moreover, we analyzed the M1 and M2 signature genes Irf4, Irf5, and Arg. ${ }^{21}$ No differences in bioenergetics capacities (Supplementary Figure 1A-D) and Irf4, Irf5, or Arg1 gene expression (Supplementary Figure 1E) was observed in these assays. Together, these findings suggest that miR-511-3p does not directly modulate M1/M2 polarization, consistent with previous reports, ${ }^{8,16}$ although it may influence the functions of alternatively activated macrophages. ${ }^{5}$

We next examined whether miR-511-3p affects Tlr4 expression in our in vitro M1 and M2 cell cultures. $\mathrm{MrCl}^{-1-}$ displayed lower levels of Tlr4, which was shown previously to be indirectly upregulated by transfection of pre-miR-511 in monocytes. ${ }^{6}$ As CD206 is more highly expressed in macrophages than monocytes, we focussed on the former cells. Overexpression of miR-511-3p in immortalized macrophages enhanced the levels of Tlr4 by $50 \%$ (Figure 6a). Upon LPS stimulation of the cells, Il6, Tnfa, and $\mathrm{Ccl} 2 \mathrm{mRNA}$ levels increased by $50 \%$ or more compared with unstimulated cells (Figure 6b). When faecal supernatant was added to the cells, Tnfa and Ccl2 levels were also significantly increased in cells overexpressing miR-511-3p (Figure 6c). Conversely, immortalized macrophages in which miR-511-3p levels were knocked down showed reduced levels of Tlr4 at baseline (Figure 6d) and reduced levels of Il6, Tnfa, and $\mathrm{Ccl} 2$ upon LPS (Figure 6e) or faecal supernatant stimulation (Figure 6f). These data suggest that miR-511-3p influences macrophage responses to LPS by regulating Tlr4 levels.

\section{miR-511-3p in human Crohn's disease patients}

Anti-TNF treatment is the end stage therapy in CD, and previous work suggests that anti-TNF treatment in $\mathrm{CD}$ patients induces the recruitment of regulatory CD206-positive macrophages that contribute to local healing in patients; such macrophages were not found in patients without mucosal healing. ${ }^{22,23}$ As our data demonstrate that miR-511-3p has a role in intestinal inflammation in mouse models, we examined the role of miR-511-3p in CD patients treated with the antiTNF adalimumab.

We first determined miR-511-3p levels in blood monocytederived macrophages that were either activated with IFN $\gamma$ or IL-4, or were matured in the presence of anti-TNF treatment, i.e., macrophages produced in a mixed lymphocyte reaction after adding anti-TNF. ${ }^{23}$ Consistent with previous studies, ${ }^{5}$ the human miR-511-3p sequence was undetectable in human M1 macrophages. Conversely, miR-511-3p levels were increased in M2 macrophages as well as in anti-TNF-treated macrophages (Figure 7a). We subsequently determined the levels of miR511-3p in biopsies of eight CD patients before and after an 8 -week treatment with anti-TNF (adalimumab; patient characteristics can be found in Supplementary Table II). Biopsies were taken from both inflamed and non-inflamed areas. After 

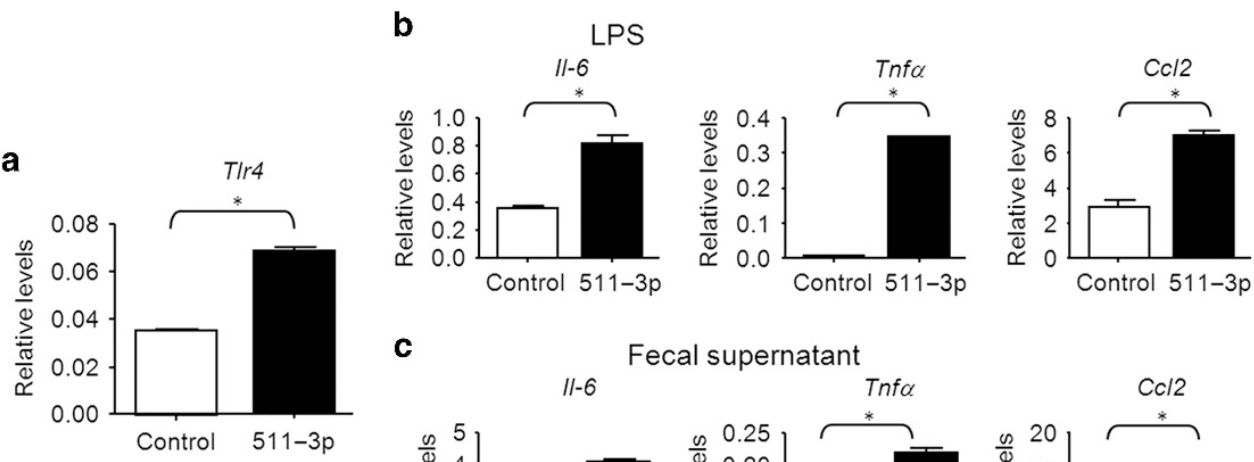

\section{C}
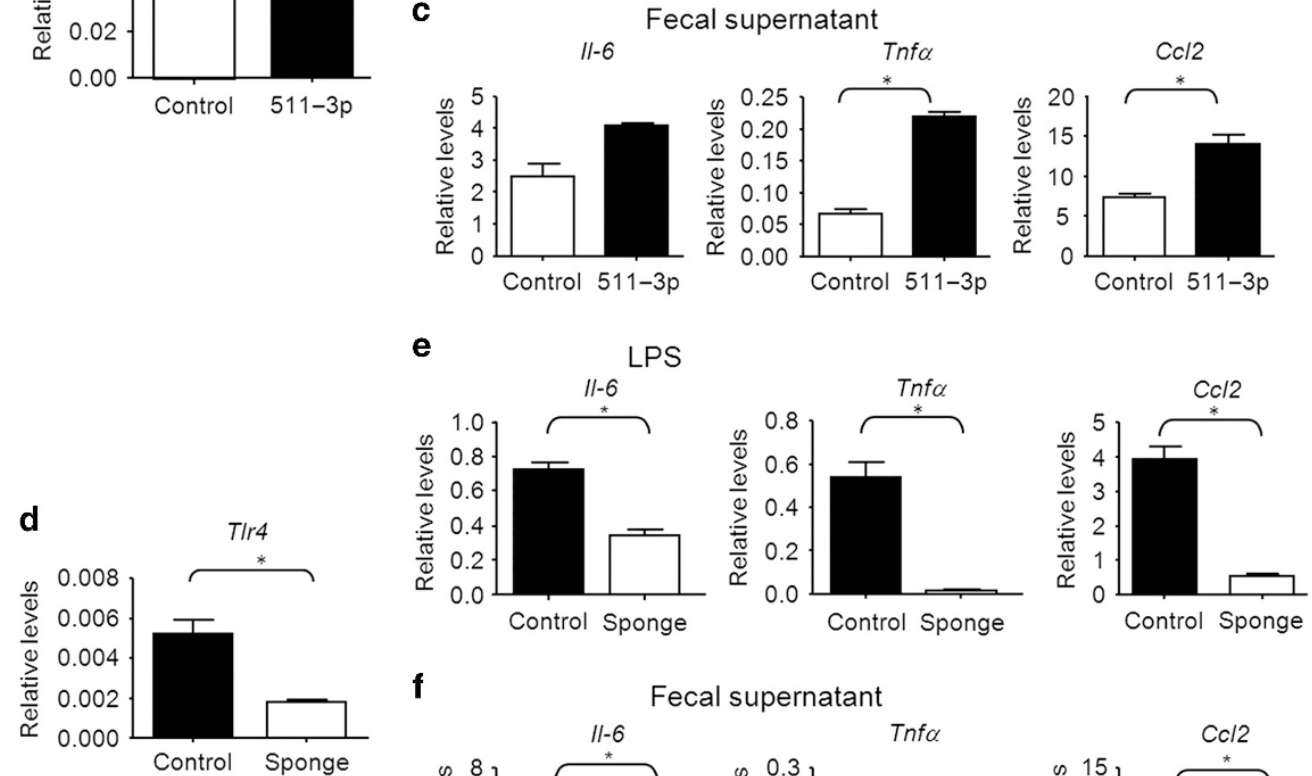

f
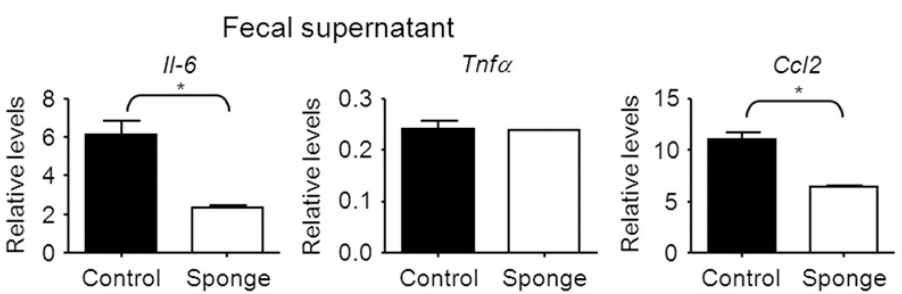

Figure 6 MiR-511-3p negatively regulates TLR4 expression levels. Macrophage cell lines transduced to overexpress miR-511-3p (black bars) or transduced with control vector (white bars) were cultured for 2 days with interleukin (IL)-4 (M2). (a) Subsequently, levels of TIr4 mRNA was determined. Matured M2 macrophages were also stimulated with LPS (b) or faecal supernatant (c) for $4 \mathrm{~h}$. II-6, Tnfo, and Ccl2 levels were determined using quantitative polymerase chain reaction (qPCR) on cell lysates. (d) Macrophage cell lines transduced to knockdown miR-511-3p (white bars) or transduced with scrambled control vector (black bars) were cultured for 2 days with IL-4, after which levels of T/r4 mRNA were determined. Macrophage cell lines matured into M2 macrophages were stimulated with LPS (e) or faecal supernatant (f) for $4 \mathrm{~h}$. II-6, Tnfo, and Cc/2 levels were determined using qPCR. These data are representative of three independent experiments, error bars indicate the s.d. A $t$-test was used for statistical analysis. ${ }^{\star} P<0.05$.

8 weeks of treatment, five patients responded to therapy according to their improved $\mathrm{CD}$ endoscopic index severity score (CDEIS), blood CRP determination, CDAI score, and IBDQ score, whereas three patients did not respond to treatment. Out of the 16 paired measured biopsies, we found that on treatment, miR-511-3p levels decreased in 11 measurements and increased in 5 (Figure 7b). Notably, baseline levels of miR-511-3p were higher in non-inflamed tissue of responders compared with that of non-responders, whereas at 8 weeks responders and non-responders had similar levels of miR-511-3p (Figure $7 \mathbf{b}$ ). In $80 \%$ of the responders, miR-511-3p levels decreased in non-inflamed tissue (Figure 7b); on average, expression was reduced by fourfold in these tissues. Such changes were less pronounced in non-responders (Figure 7c). This expression pattern may be due to higher level of expression of miR-511-3p in responders at the start of therapy. Consistent with this notion, a higher percentage of CD206-positive macrophages prior infliximab treatment was found in patients responding to anti-TNF in an earlier study. ${ }^{23}$ Together, these data suggest that a decrease in expression of miR-511-3p in non-inflamed tissues may be associated with response to anti-TNF treatment, although, because of the limited number of patients included (three to five in each group), we could not find differences that reached statistical significance.

\section{DISCUSSION}

CD206 is an endocytic receptor expressed by maturing monocytes and macrophages in the mouse colonic mucosa. In this study, we show that mice lacking the gene for CD206, Mrc1, display reduced sensitivity to DSS-induced colitis, a phenotype not mediated by the absence of CD206 itself but by the decreased expression of miR-511-3p, a miRNA co-regulated with $M r c 1$ gene expression. ${ }^{5,16}$ We show that 


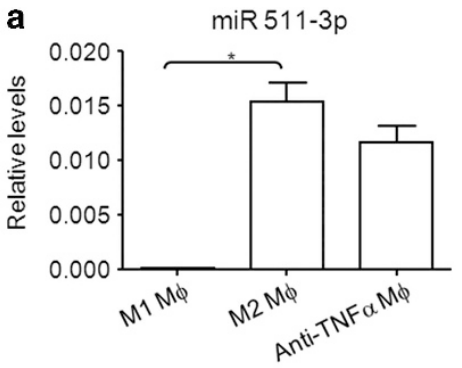

b
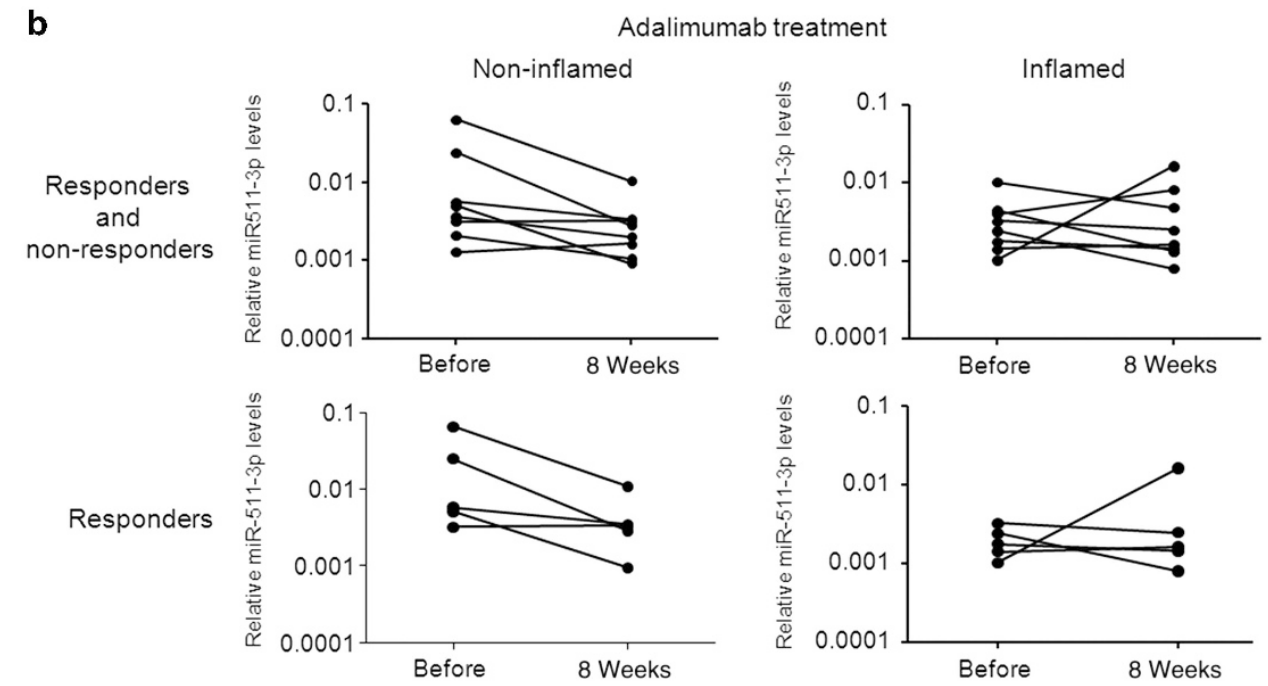

Non-responders
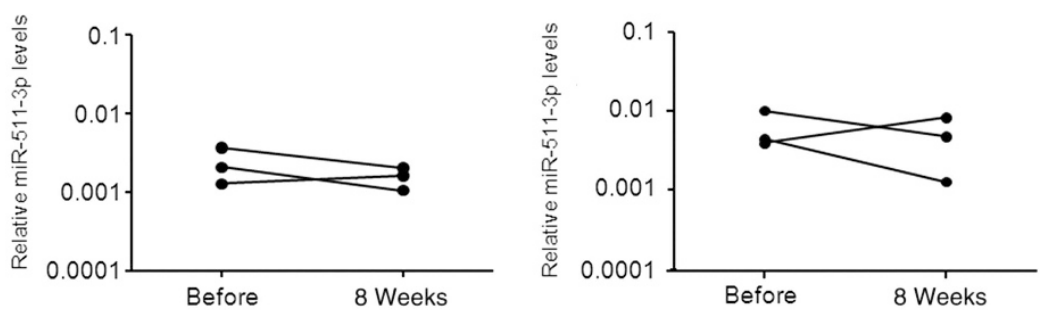

C Fold reduction in miR-511-3p levels

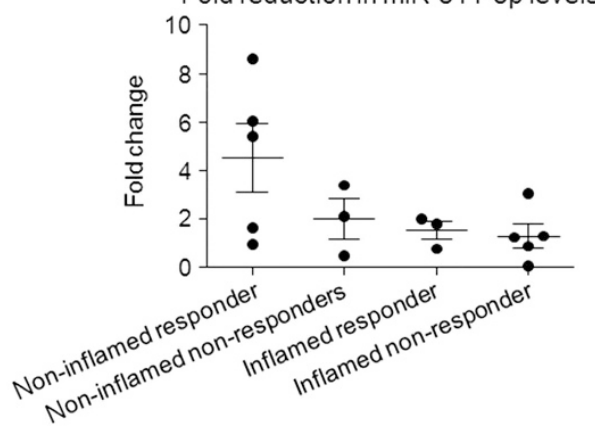

Figure 7 miR-511-3p levels in Crohn's disease (CD) patient biopsy material. (a) miR-511-3p levels were determined in healthy donor human monocytederived macrophages cultured with interferon- $\gamma$ (IFN $\gamma$; M1 m $\phi$ ), interleukin (IL)-4 (M2 m $\phi)$, or macrophages produced by a mixed lymphocyte reaction cultured with adalimumab (tumor necrosis factor- $\alpha$ (TNF- $\alpha, \mathrm{m} \phi)$. Bars represent relative miR-511-3p levels. Error bars indicate the s.d. A $t$-test was used for statistical analysis. ${ }^{\star} P<0.05$. (b) miR-511-3p levels were determined in biopsies from patients before and after adalimumab treatment. Biopsies were taken from both inflamed and non-inflamed areas and patients were categorized into treatment responders and non-responders. (c) Fold change of miR511-3p levels after 8 weeks of adalimumab treatment for inflamed and non-inflamed areas in treatment responders and non-responders. A $t$-test was used for statistical analysis. ${ }^{*} P<0.05$.

$\mathrm{MrCl}^{-1-}$ macrophages have reduced levels of miR-511-3p as well as reduced levels of Tlr4 and that miR-511-3p enhances the macrophage response to LPS by controlling
Tlr4 levels. This report is the first to demonstrate that macrophage miR-511-3p expression mediates the course of experimental colitis. 
$\mathrm{MrCl}^{-1-}$ mice were generated by inserting an enhanced green fluorescent protein (eGFP) complementary DNA (cDNA) at the CD206 start codon; the eGFP has its own stop codon, which abolishes CD206 expression. ${ }^{24}$ Several studies using $\mathrm{Mrcl}^{-1-}$ mice suggest that CD206 is involved in ligand binding and subsequent responses. ${ }^{10,24-26}$ However, it is important to revisit these results, given that these effects may rather be caused by the reduced levels of miR-511-3p, which affects expression of other pattern recognition receptors. ${ }^{1}$ In general, this warrants caution in the interpretation of studies using knockout mice, as phenotypes could be mediated by miRNAs hosted in the gene locus. $\mathrm{MrCl}^{-1-}$ mice have delayed clearance of serum lysosomal hydrolases, but otherwise appear healthy. ${ }^{24}$ In infection studies with different fungi or Leishmania, these mice showed no differences in disease severity compared with WT mice; ${ }^{27-29}$ however, $\mathrm{Mrcl}^{-1-}$ mice were protected in a crescentic glomerulonephritis model because of a requirement for CD206 in mediating FcR-mediated functions. ${ }^{26}$

CD206 is involved in the presentation of mannosylated proteins to naive $\mathrm{T}$ cells and, as $\mathrm{CD}$ patients appear to have lost tolerance to mannan-exposing fungi, it is plausible that CD206 has a role in this process. ${ }^{30,31}$ In the context of colitis, mannan, a major ligand of the CD206 that blocks its function, has been shown to reduce inflammation in 2,4,6-trinitrobenzenesulfonic acid colitis. ${ }^{32}$ CD206 has a role in microbial binding and, furthermore, has been shown to regulate subsequent cytokine responses and intracellular microbial killing (reviewed in $\mathrm{Gazi}^{33}$ ). As CD206 does not contain any known signaling motifs, it is likely that the CD206 is located in a signaling synapse enabling proximity by binding. This functionality would enable interaction with other pattern recognition receptors without the need for $\mathrm{CD} 206$ to induce its own signaling. In this study, we show that $\mathrm{MrCl}^{-1-}$ mice are less sensitive to experimental colitis compared with WT, whereas the in vivo application of a CD206-neutralizing antibody or an antagonist had no effect. We observed that $\mathrm{MrCl}^{-1-}$ BMDMs have a reduced response to LPS associated with reduced levels of TLR4, an effect that may be explained by the reduction of miR-511-3p. Consistently, blocking the miR511 precursor (which produces both $3 p$ and $5 p$ mature strands) in human blood monocyte-derived DCs reduced TLR4 levels in these cells. ${ }^{6}$ We now show that specifically the functional miRNA strand, miR-511-3p, ${ }^{5,14,15}$ regulates TLR4 RNA levels in mouse macrophages and likely controls the course of colitis.

Although miR-511 was shown to regulate TLR4 expression, ${ }^{6}$ this precursor miR-511 strand is increasingly recognized as a non-functional miRNA. ${ }^{5,14,15}$ On the other hand, TLR4 is not a predicted target of miR-511-3p according to Targetscan; therefore, it is likely that miR-511-3p downregulates a negative regulator of TLR expression. miRNAs are often fine-tuning regulators of several targets and hundreds of miR-511-3p targets can be found in databases; it is possible that other targets also have a role in inflammation and further studies are needed to determine this.
The role of TLR4 has been studied extensively in experimental and human IBD. In the intestine, both immune cells and intestinal epithelial cells (IEC) express TLR4, which is important in maintaining barrier function and an optimal IEC proliferation. ${ }^{34}$ In human studies, TLR4 is clearly upregulated during inflammation and various single nucleotide polymorphism in the TLR4 gene have been implicated in CD. ${ }^{7}$ Mouse colitis studies show conflicting results of susceptibility and resistance in $T l r 4^{-1-}$ mice. ${ }^{35}$ Upon DSS injury, TLR4 ${ }^{-1-}$ mice have aggravated disease, ${ }^{36,37}$ caused by increased epithelial damage due to decreased epithelial proliferation. ${ }^{37}$ Several other studies show that TLR4 antagonists ameliorate inflammation in DSS-treated mice by reducing the inflammatory cell influx and reducing their pro-inflammatory cytokine and chemokine production. ${ }^{38-40}$ In our experiments with $\mathrm{MrCl}^{-1-}$ mice, we found that TLR4 expression is reduced in macrophages. We also observed reduced influx and cytokine production during DSS-induced inflammation, supporting the idea that TLR4 on inflammatory macrophages is detrimental during intestinal inflammation. As we now provide in vitro and in vivo data that miR-511-3p positively regulates TLR4 levels on macrophages, this may represent a therapeutic target for treating IBD patients.

It was shown earlier that the upregulation of CD206, and miR-511-3p, is associated with the alternative activation of protumoral tumor associated macrophages. ${ }^{5}$ Similarly, we observed upregulated expression of CD206 along with miRNA-511-3p in human and mouse M2-polarized macrophages. We show that in the mouse colonic mucosa-maturing monocytes and macrophages express CD206 with only the later containing miR-511-3p. It is interesting to note that in IBD mucosa, the efficacy of treatment with TNF-neutralizing medication is associated with an enhanced expression of CD206 on regulatory macrophages found in responsive treated mucosa. ${ }^{22,23}$ In fact, it is suggested that the potential of anti-TNF antibody ligation to give rise to CD206-positive regulatory macrophages is an explanation for the clinical effect of anti-TNF medication in IBD. ${ }^{22,23}$ In our study, we analyzed the expression of miR-511-3p levels in CD patients treated with adalimumab, and correlated expression to the response rate of this treatment. We confirmed that anti-TNF-treated macrophages upregulated miR-511-3p expression in vitro. We did not find significant differences in miR-511-3p levels between responders and non-responders before or at 8 weeks into treatment. Overall, we did observe reduced miR-511-3p levels during anti-TNF treatment, which appeared more pronounced in non-inflamed biopsies of responding patients. However, patient numbers were not high enough for a sufficient power to achieve statistical significance. Whether miR-511-3p could serve as a predictive biomarker for response to anti-TNF treatment in IBD will be a subject of future studies.

A recent study by Puimège et al. ${ }^{41}$ suggested that Tnfrsfla is a naturally occurring target of miR-511 passenger strand miR511-5p. The authors found that selective miR-511-5p inhibition decreases survival of lethally TNF $\alpha$-treated mice. This observation may suggest that miR-511-3p and miR-511-5p may have 
complementary and/or opposite functions to regulate macrophage activation and inflammatory responses. Thus, while miR-511-3p promotes expression of pro-inflammatory cytokines in macrophages, miR-511-5p might downregulate TNFR1 and thus attenuate inflammation. ${ }^{41}$ However, experiments using reporter vectors to measure miRNA activity, as well as miRNA expression analysis using $\mathrm{qPCR}$, have shown that miR-511-3p is the active and more expressed strand of premiR-511 in IL-4-polarized macrophages, tissue macrophages and tumor-associated macrophages. ${ }^{5}$ In agreement with these initial findings, the current version of the miRNA registry miRBASE (v21, year 2015) shows that out of the 6229 reads mapped in the mouse miR-511 locus, 129 reads correspond to the $-5 p$ strand and 5,606 reads correspond to the $-3 p$ strand. ${ }^{42}$ Together, these data suggest that miR-511-3p is the most abundant, and likely only active, strand of pre-miR-511. However, it cannot be excluded that in selected cell types and/or differentiation/activation states, miR-511-5p may function as the active product of pre-miR-511. Further studies are, therefore, warranted to understand whether miR-511-5p and miR-511-3p differentially modulate inflammatory responses in different contexts, and the underlying mechanism of strand selection during pre-miR-511 processing.

To avoid excessive inflammation while enabling protection against pathogens, inflammatory responses to LPS need to be tightly regulated, particularly in the intestine. We found that miR-511-3p is an unexpected regulator of this process, and as such is a potential mediator of inflammation in the colon and the course of IBD.

\section{METHODS}

Mice. Breeding colonies of C57BL/6 and C57BL/6/ $\mathrm{Mrcl}^{-1-}$ mice, kindly provided by Dr Michel Nussenzweig, ${ }^{24}$ were housed and maintained under specific pathogen-free conditions in our animal facility at the Academic Medical Center in Amsterdam. Mice were kept and handled in accordance with the guidelines of the Animal Research Ethics Committee of the University of Amsterdam.

Colitis experiments. Mice were sex-matched male or female and between 8 and 12 weeks of age at the time of study. For DSS colitis experiments $1.5 \%$ (w/v) DSS (TdB Consultancy, Uppsala, Sweden) was added to the drinking water for 7 days. Fresh DSS solutions were prepared daily. Mice received drinking water for the following days of the experiments. In the experiment using anti-CD206 antibody (clone 5D3, Serotec, Puchheim, Germany) and IgG2a isotype control antibody (produced in-house), $1 \mathrm{mg}$ was injected intraperitoneally on days 3 and 10 of the experiment. In the experiment using mannan to antagonize CD206, Saccharomyces cerevisiae mannan (Sigma, Zwijndrecht, The Netherlands) was dissolved and $1 \mathrm{mg}$ was given daily by oral gavage. One milligram per day was previously shown to suffice to neutralize CD206 and induce immune-modulating effects in mice for similar carbohydrates. ${ }^{43}$ Mice were pre-treated with mannan in the drinking water for 14 days before inducing DSS colitis. Mannancontaining drinking water was refreshed daily. Body weights were recorded daily. At the end of the study, mice were killed and organs collected. Wet weights of the spleen and colon were recorded together with the total length of the colon. Colon weight per $6 \mathrm{~cm}$ was used as a disease parameter. Faeces were scored as follows: (0) normal faeces, (1) soft pellets, (2) thin faeces, (3) watery diarrhea, and (4) bloody diarrhea. The score indicators for intestinal inflammation are summarized in Supplementary Table I.
Histology. The longitudinally divided colons were rolled, fixed in $4 \%$ formalin, and embedded in paraffin for routine histology. An experienced pathologist evaluated formalin-fixed hematoxylin tissue sections microscopically, in a blinded manner. Colons were evaluated and graded from 0 to 4 as an indication of incidence and severity of inflammatory lesions based on the extent of the area involved, the number of follicle aggregates, edema, fibrosis, hyperplasia, erosion/ ulceration, crypt loss and infiltration of granulocytes, and mononuclear cells as indicated in Supplementary Table I. The total inflammation score was calculated as the average score of the above.

Fluorescent immunohistochemistry. Sections were acetone-fixed and blocked in $10 \%$ normal rabbit serum for $15 \mathrm{~min}$. Slides were drained and incubated with purified monoclonal antibody 5D3 (AbD Serotec) or the rat isotype control at $10 \mu \mathrm{g} \mathrm{ml}^{-1}$ for $1 \mathrm{~h}$. After washing in hosphate-buffered saline (PBS), the sections were incubated with Alexa 568-labeled goat anti-rat IgG (Molecular Probes, Leiden, The Netherlands) for $1 \mathrm{~h}$. After washing, the sections were incubated with fluorescein isothiocyanate -conjugated anti-mouse CD11b for $1 \mathrm{~h}$. Slides were washed and mounted in a medium containing 4,6-diamidino-2-phenylindole (Vectashield, Burlingame, CA).

Measurements of colonic cytokines. Frozen colonic tissue was homogenized on ice in Greenberger Lysis Buffer $(150 \mathrm{mM} \mathrm{NaCl}$, $15 \mathrm{mM}$ Tris, $1 \mathrm{mM} \mathrm{MgCl} \cdot 6 \mathrm{H}_{2} \mathrm{O}, 1 \mathrm{mM} \mathrm{CaCl}_{2}$, and $1 \%$ Triton) with protease inhibitor cocktail from Roche (Woerden, The Netherlands; 11697498001), pH 7.4, diluted 1:1 with PBS. Cytokine levels of interleukin (IL)-12, interferon- $\gamma$ (IFN $\gamma$ ), TNF- $\alpha$ (tumor necrosis factor- $\alpha$ ), IL-10, CCL2, and IL-6 were measured in homogenates using ELISA kits (R\&D, Abingdon, UK) according to the manufacturer's protocol.

Lentiviral vector production and transduction. Vesicular stomatitis virus-pseudotyped, third-generation lentiviral vectors (LVs) were produced by transient four-plasmid co-transfection into 293T cells and concentrated using ultracentrifugation, as described. ${ }^{44}$ Expression titers of LVs expressing fluorescent proteins were determined on 293T cells by limiting dilution assays. LV stocks were stored at $-80^{\circ} \mathrm{C}$.

Primary mouse macrophage culture. Bone marrow cells were isolated from tibiae and femurs of C57BL/6 and C57BL/6/Mrcl ${ }^{-1-}$ mice. Cells were cultured for 8 days in RPMI-1640 (Invitrogen) supplemented with $100 \mathrm{U} \mathrm{ml}^{-1}$ penicillin/streptomycin (GIBCO Invitrogen), $2 \mathrm{mM}$ glutamine (Invitrogen, Bleiswijk, The Netherlands), $10 \%$ heat-inactivated fetal calf serum (FCS; GIBCO Invitrogen, Merelbeke, Belgium), and 15\% (vol/vol) L929-cell-conditioned medium as a source of macrophage colony-stimulating factor. Fresh medium was added on day 4 after isolation. On day 7 after isolation, cells were harvested with PBS containing $4 \mathrm{mg} \mathrm{ml}^{-1}$ lidocaine- $\mathrm{HCl}$, plated at $10^{7}$ macrophages in $10-\mathrm{cm}^{2}$ culture plates and differentiated into M2 macrophages by culture for 2 days in a medium containing $40 \mathrm{ng} \mathrm{ml}^{-1}$ IL-4 (Peprotech, London, UK).

BMDM cell lines. We used the following mouse BMDM cell lines, which have been described recently in ${ }^{8}(1)$ miR-511-3p cell line, which was obtained by stably transducing immortalized BMDMs ${ }^{8}$ with a LV overexpressing miR-511-3p. ${ }^{5}$ (2) miR-control cell line, which was obtained by stably transducing immortalized $\mathrm{BMDMs}^{8}$ with a LV expressing a mutated form of miR-511-3p. ${ }^{5}$ (3) miR-511-3p sponge cell line, used to knockdown miR-511-3p. This cell line was obtained by stably transducing immortalized $\mathrm{BMDMs}^{8}$ with a $\mathrm{LV}$ expressing eight tandem sequences perfectly complementary to miR-511-3p. ${ }^{8}$ The robust overexpression of miR-511-3p target sequences detectably decreases endogenous miR-511-3p levels likely through a ' $m i R$ sponge effect'. ${ }^{8,19}$ (4) miR-control sponge cell line, obtained by transducing immortalized $\mathrm{BMDMs}^{8}$ with a LV expressing scrambled miR-511-3p antisense sequences. ${ }^{8}$ Transduced immortalized BMDM lines were cultured in RPMI-1640 (Invitrogen) supplemented with $100 \mathrm{U} \mathrm{ml}^{-1}$ 
penicillin/streptomycin (GIBCO Invitrogen), $2 \mathrm{mM}$ glutamine (Invitrogen), 10\% FCS (GIBCO Invitrogen), and 20\% (vol/vol) L929cell-conditioned medium as a source of macrophage colony-stimulating factor. Before experiments, 107 macrophages were cultured in $10-\mathrm{cm}^{2}$ culture plates and differentiated into M1 or M2 macrophages by culturing them for 2 days in a medium containing $50 \mathrm{ng} \mathrm{ml}^{-1}$ IFN $\gamma$ (Peprotech) or $40 \mathrm{ng} \mathrm{ml}^{-1} \mathrm{IL}-4$ (Peprotech), respectively.

Determination of macrophage metabolic activity. One day prior to analysis, macrophage cell lines were plated in XF96 cell culture microplates (Bioscience, Leiden, The Netherlands) and polarized using $50 \mathrm{ng} \mathrm{ml}^{-1} \mathrm{IFN} \gamma$ (Peprotech) and $10 \mathrm{ng} \mathrm{ml}^{-1}$ LPS for differentiation into M1 macrophages or $40 \mathrm{ng} \mathrm{ml}^{-1} \mathrm{IL}-4$ (Peprotech) for differentiation into M2 macrophages. After $24 \mathrm{~h}$, the culture medium was replaced by unbuffered DMEM (Sigma) supplemented with $25 \mathrm{mM}$ glucose and $200 \mathrm{mM}$ glutamine. Cells were incubated at $37^{\circ} \mathrm{C}$ without $\mathrm{CO}_{2}$ for $1 \mathrm{~h}$ to allow the medium temperature and $\mathrm{pH}$ to reach equilibrium. Subsequently, the oxygen consumption rate (OCR) and extracellular acidification rates were measured using a Seahorse Extracellular Flux (XF) Analyzer (Seahorse Bioscience, Copenhagen, Denmark), following the manufacturer's instructions. Basal OCR and extracellular acidification rate as well as response to sequential incubation with oligomycin $(15 \mu \mathrm{M})$, carbonyl cyanide-p-trifluoromethoxyphenylhydrazone (FCCP, $1.5 \mu \mathrm{M}$ ), and antimycin A $(2.5 \mu \mathrm{M})$ with rotenone $(1.25 \mu \mathrm{M})$ were recorded. Oligomycin is an ATP synthase inhibitor and differentiates oxygen consumption that is used for ATP synthesis from proton leak across the inner mitochondrial membrane. FCCP uncouples the proton transport from ATP synthesis, resulting in maximal oxygen consumption without mitochondrial ATP generation, reflecting the maximal respiratory capacity of the cells. The difference between maximal OCR (after addition of FCCP) and basal OCR is the mitochondrial spare respiratory capacity. Antimycin A and rotenone mix inhibits mitochondrial complex I and III, which interferes with the use of NADH for ATP synthesis. The antimycin A and rotenone-insensitive OCR reflects nonmitochondrial respiration, which was used to normalize OCR and extracellular acidification rate data.

LPS and faecal content stimulation. Overall, $1.5 \times 10^{6}$ macrophages were plated in six-well tissue culture plates with RPMI containing $50 \mathrm{IU} \mathrm{ml}{ }^{-1}$ penicillin, $50 \mu \mathrm{ml}^{-1}$ streptomycin, and $2 \mathrm{mML}$-glutamine at $37^{\circ} \mathrm{C}$ in $5 \% \mathrm{CO}_{2}$. After $2 \mathrm{~h}$, once macrophages had adhered, LPS $\left(500 \mathrm{ng} \mathrm{ml}^{-1}\right)$ or faecal supernatants were added and incubated with the cells for $4 \mathrm{~h}$. Faecal supernatant was produced by suspending the contents of a mouse cecum in $50 \mathrm{ml}$ PBS, which was subsequently filtered over a $40-\mu \mathrm{m}$ filter and frozen at $-20^{\circ} \mathrm{C}$ and used in a $1: 1,000$ dilution. After 24-h incubation, no growth of microorganisms was found in the wells and no cell death was observed by microscopic check for floating cells. Supernatants of cell stimulation were kept at $-20^{\circ} \mathrm{C}$ till the determination of cytokine levels using ELISA kits (R\&D) according to the manufacturer's protocol. Cells were stored in Tripure (Roche) at $-80^{\circ} \mathrm{C}$ for RNA isolation.

Quantitative PCR. Tlr2, Tlr4, Mcp1, Il6, Tnf $\alpha$, and Ccl2 transcript levels were determined in macrophage lysates using quantitative polymerase chain reaction (qPCR). Two micrograms of RNA was treated with DNase (Promega, Leiden, The Netherlands) and reversetranscribed with oligo (dT) and random primers and Superscript II reverse transcriptase (Invitrogen). One microliter of the complementary DNA (cDNA) solution was subjected to real-time qPCR in a Roche Lightcycler PCR, using Lightcycler SYBR Green (Roche). Expression levels were analyzed using the LinRegPCR software and normalized for housekeeping genes Hprt and Gapdh.

To determine the levels of miR-511-3p in cells and tissue, cDNA was produced using the Universal cDNA synthesis kit II (Exiqon). Subsequently, $1 \mu \mathrm{l}$ of cDNA solution was subjected to real-time qPCR in a Roche Lightcycler PCR as above, using ExiLENT SYBR Green master mix (Exiqon, Vedbaek, Denmark). Expression levels were analyzed using the LinRegPCR software and normalized for human tissues using SNORD38B (a small nuclear RNA) and for mouse samples using $5 S$.

Human macrophage culture. Human monocytes were obtained from normal blood donor buffy coats by Ficoll-Paque PLUS (GE Healthcare, Breda, The Netherlands) separation. Monocytes were adhered to tissue culture plastic for $90 \mathrm{~min}$, after which unattached cells were removed by washing with PBS. Macrophages were obtained by culturing monocytes in IMDM containing $100 \mathrm{U} \mathrm{ml}^{-1}$ penicillin/ streptomycin (GIBCO Invitrogen), 2 mM glutamine (Invitrogen), 10\% FCS (GIBCO Invitrogen) supplemented with $50 \mathrm{ng} \mathrm{ml}^{-1}$ IFN $\gamma$ (Pepro Tech; M1) or $40 \mathrm{ng} \mathrm{ml}^{-1}$ IL-4 $\gamma$ (Pepro Tech; M2) for 4 days. Cells were detached using $10 \mathrm{mM}$ EDTA and suspended in 48-well tissue culture plastic at $5 \times 10^{5}$ cells per well. Human macrophages treated with anti-TNF antibody (adalimumab) were produced as described. ${ }^{22}$ Briefly, PBMCs from two healthy donors were cultured in a 1:1 ratio in RPMI 1640 culture medium. After $48 \mathrm{~h}$, cells were treated with $10 \mu \mathrm{g} \mathrm{ml}^{-1}$ anti-TNF antibody (adalimumab) for 7 days. Adalimumab-treated macrophages were isolated using CD14 microbeads according to the manufacturer's protocol (Miltenyi Biotech, Leiden, The Netherlands).

Isolation and sorting of colonic lamina propria cells. Colons of mice were removed and cleaned from fat and faeces. Colons were opened longitudinally, washed in PBS, and cut into $0.5-\mathrm{cm}$ pieces. Colon pieces were washed several times by vigorous shaking in Hanks' balanced salt solution (HBSS) (Invitrogen) with 2\% FCS (GIBCO Invitrogen). Tissue pieces were incubated shaking for $20 \mathrm{~min}$ at $37^{\circ} \mathrm{C}$ in calcium/ magnesium-free HBSS supplemented with $2 \%$ FCS and 5 mM EDTA (Sigma). Tissues were washed with ice-cold PBS and subsequently cut into $0.1-\mathrm{cm}$ pieces. Tissue pieces were incubated for $40 \mathrm{~min}$ under vigorous shaking at $37^{\circ} \mathrm{C}$ with calcium/magnesium-free HBSS supplemented with $2 \%$ FCS, $5 \mathrm{mg} \mathrm{ml}^{-1}$ Liberase Blendzyme (Roche), and $10 \mu \mathrm{g} \mathrm{ml}^{-1}$ DNAse (Roche). After complete digestion, the cell suspension was passed through a $100-\mu \mathrm{m}$ cell strainer.

Cells were stained for $30 \mathrm{~min}$ on ice in PBS containing $1 \%$ FCS, $0.5 \mu \mathrm{g} \mathrm{ml}^{-1}$ anti-CD16/CD32 (FCblock, BD Pharmingen, Breda, The Netherlands), PerCP-conjugated anti-CD11c (Biolegend, Uithoorn, The Netherlands), APC-conjugated anti-Ly6C (eBioscience, Vienna, Austria), APC-Cy7-conjugated anti-CD45 (Biolegend), PE-Cy7-conjugated anti-MHCIIa (Biolegend), fluorescein isothiocyanate-conjugated anti-Ly6G (Biolegend), PE-conjugated anti-CD64 (Biolegend), and Alexa700-conjugated anti-CD11c (eBioscience). Cells were washed two times in PBS with $2 \%$ FCS. Cells were resuspended in $2 \mathrm{ml}$ PBS with $2 \%$ FCS and $40 \mu \mathrm{ml}^{-1}$ RNAse inhibitor (Ribolock, Thermo Scientific, Breda, The Netherlands). Before sorting, 4,6-diamidino-2-phenylindole Nucleic Acid Stain (Invitrogen) was added at a concentration of $0.5 \mathrm{gg} \mathrm{ml}^{-1}$. Cells were sorted using FACS Aria cell sorter (BD Bioscience), and monocyte, macrophage, and DC populations were collected in PBS with $2 \%$ FCS and $20 \mu \mathrm{ml}^{-1}$ Ribolock. RNA was isolated using a miRNeasy micro kit (Qiagen, Venlo, The Netherlands) following the total RNA, including the miRNA isolation protocol.

Biopsies from CD patients. Crohn's disease (CD) patients scheduled for colonoscopy because of clinical symptoms suggestive of active disease all gave informed consent before participation. The study protocol was approved by the Academic Medical Centre's ethical committee. Patient characteristics are given in Supplementary Table II.

Patients were included when active disease was observed during colonoscopy and/or imaging by CD disease activity index (CDAI), and new remission induction therapy was initiated. Before colonoscopy, blood for c-reactive protein determination, the CDAI, and IBD questionnaire (IBDQ) were obtained from every patient. During colonoscopy, the CDEIS was obtained and biopsies collected from inflamed and non-inflamed regions of the intestine. After 8 weeks, 
patients again completed the CDAI and IBDQ, and donated blood. Furthermore, a second colonoscopy was performed after 8 weeks of induction therapy including scoring of the CDEIS and collection of biopsies of inflamed and non-inflamed regions, when applicable.

Response to the induction therapy was determined primarily by the changes in CDEIS and secondly by c-reactive protein after 8 weeks of therapy. Furthermore, CDAI and IBDQ scores were obtained before and after 8 weeks. The CDEIS was considered to be 'gold standard'; however, patients classified as 'responders' should show at least $20 \%$ improvement on all activity scores. Patients were classified as 'nonresponders' when $\mathrm{CD}$ activity scores remained equal or deteriorated after 8 weeks of therapy. Biopsies were completely immersed in RNAlater RNA stabilization reagent (Qiagen), and miRNA isolation was performed using the miRNeasy minikit (Qiagen) according to the manufacturer's protocol.

Statistical analysis. Data are expressed as mean \pm s.d. Statistically significant $(P<0.05)$ differences between two groups were evaluated using a two-tailed unpaired $t$-test, when comparing multiple groups a one-way analysis of variance (ANOVA) with Bonferroni multiple comparison test was used.

SUPPLEMENTARY MATERIAL is linked to the online version of the paper at http://www.nature.com/mi

\section{ACKNOWLEDGMENTS}

We thank the staff of our animal facility for care of the mice used in this study. We also thank Dr R.M. van den Wijngaard for helpful discussions and Dr Jan van den Bossche and Dr Vincent de Boer for help with the Seahorse experiments. This work was funded by an NWO VIDI grant to WJdeJ and Fonds National Suisse de la Recherche Scientifique (SNSF grant 31003A143978) to MDP.

\section{DISCLOSURE}

WJdeJ receives research grants and consultancy fees from GlaxoSmithKline, Mead Johnsson Pediatric Institute, and Schwabe corporation. The remaining authors declared no conflict of interest.

(c) 2016 Society for Mucosal Immunology

\section{REFERENCES}

1. Martinez-Pomares, L. The mannose receptor. J. Leukoc. Biol. 92, 1177-1186 (2012).

2. Gordon, S. \& Martinez, F.O. Alternative activation of macrophages: mechanism and functions. Immunity 32, 593-604 (2010).

3. Burgdorf, S., Kautz, A., Bohnert, V., Knolle, P.A. \& Kurts, C. Distinct pathways of antigen uptake and intracellular routing in CD4 and CD8 T cell activation. Science 316, 612-616 (2007).

4. Burgdorf, S., Lukacs-Kornek, V. \& Kurts, C. The mannose receptor mediates uptake of soluble but not of cell-associated antigen for crosspresentation. J. Immunol. 176, 6770-6776 (2006).

5. Squadrito, M.L. et al. miR-511-3p modulates genetic programs of tumorassociated macrophages. Cell Rep. 1, 141-154 (2012).

6. Tserel, L. et al. MicroRNA expression profiles of human blood monocytederived dendritic cells and macrophages reveal miR-511 as putative positive regulator of Toll-like receptor 4. J. Biol. Chem. 286, 26487-26495 (2011).

7. Cario, E. Toll-like receptors in inflammatory bowel diseases: a decade later. Inflamm. Bowel Dis. 16, 1583-1597 (2010).

8. Squadrito, M.L. et al. Endogenous RNAs modulate microRNA sorting to exosomes and transfer to acceptor cells. Cell Rep. 8, 1432-1446 (2014).

9. Martinez-Pomares, L. et al. Analysis of mannose receptor regulation by IL-4, IL-10, and proteolytic processing using novel monoclonal antibodies. J. Leukoc. Biol. 73, 604-613 (2003).

10. Heinsbroek, S.E., Taylor, P.R., Martinez, F.O., Martinez-Pomares, L., Brown, G.D. \& Gordon, S. Stage-specific sampling by pattern recognition receptors during Candida albicans phagocytosis. PLoS Pathog. 4, e1000218 (2008).
11. Miller, J.L. et al. The mannose receptor mediates dengue virus infection of macrophages. PLoS Pathog. 4, e17 (2008).

12. Nguyen, D.G. \& Hildreth, J.E. Involvement of macrophage mannose receptor in the binding and transmission of HIV by macrophages. Eur. J. Immunol. 33, 483-493 (2003).

13. van de Veerdonk, F.L. etal. The macrophage mannose receptor induces IL17 in response to Candida albicans. Cell Host Microbe 5, 329-340 (2009).

14. Wong, N. \& Wang, X. miRDB: an online resource for microRNA target prediction and functional annotations. Nucleic Acids Res. 43, D146-D152 (2014).

15. Chang, H.T. et al. Comprehensive analysis of microRNAs in breast cancer. BMC Genomics 13 (Suppl 7), S18 (2012).

16. Karo-Atar, D., Itan, M., Pasmanik-Chor, M. \& Munitz, A. MicroRNA profiling reveals opposing expression patterns for miR-511 in alternatively and classically activated macrophages. J. Asthma 52, 545-553 (2014).

17. Bain, C.C. et al. Resident and pro-inflammatory macrophages in the colon represent alternative context-dependent fates of the same Ly6Chi monocyte precursors. Mucosal Immunol. 6, 498-510 (2013).

18. Cerovic, V., Bain, C.C., Mowat, A.M. \& Milling, S.W. Intestinal macrophages and dendritic cells: what's the difference? Trends Immunol. 35, 270-277 (2014).

19. Ebert, M.S. \& Sharp, P.A. MicroRNA sponges: progress and possibilities. RNA 16, 2043-2050 (2010).

20. Tavakoli, S., Zamora, D., Ullevig, S. \& Asmis, R. Bioenergetic profiles diverge during macrophage polarization: implications for the interpretation of 18 F-FDG PET imaging of atherosclerosis. J. Nucl. Med. 54, 1661-1667 (2013).

21. Lawrence, T. \& Natoli, G. Transcriptional regulation of macrophage polarization: enabling diversity with identity. Nat. Rev. Immunol. 11, 750-761 (2011).

22. Vos, A.C., Wildenberg, M.E., Duijvestein, M., Verhaar, A.P., van den Brink, G.R. \& Hommes, D.W. Anti-tumor necrosis factor-alpha antibodies induce regulatory macrophages in an Fc region-dependent manner. Gastroenterology 140, 221-230 (2011).

23. Vos, A.C. et al. Regulatory macrophages induced by infliximab are involved in healing in vivo and in vitro. Inflamm. Bowel Dis. 18, 401-408 (2012).

24. Lee, S.J. et al. Mannose receptor-mediated regulation of serum glycoprotein homeostasis. Science 295, 1898-1901 (2002).

25. Court, N. et al. Partial redundancy of the pattern recognition receptors, scavenger receptors, and c-type lectins for the long-term control of Mycobacterium tuberculosis infection. J. Immunol. 184, 7057-7070 (2010).

26. Chavele, K.M. et al. Mannose receptor interacts with Fc receptors and is critical for the development of crescentic glomerulonephritis in mice. J. Clin. Invest. 120, 1469-1478 (2010).

27. Swain, S.D., Lee, S.J., Nussenzweig, M.C. \& Harmsen, A.G. Absence of the macrophage mannose receptor in mice does not increase susceptibility to Pneumocystis carinii infection in vivo. Infect. Immun. 71, 6213-6221 (2003).

28. Lee, S.J., Zheng, N.Y., Clavijo, M. \& Nussenzweig, M.C. Normal host defense during systemic candidiasis in mannose receptor-deficient mice. Infect. Immun. 71, 437-445 (2003).

29. Akilov, O.E., Kasuboski, R.E., Carter, C.R. \& McDowell, M.A. The role of mannose receptor during experimental leishmaniasis. J. Leukoc. Biol. 81, 1188-1196 (2007).

30. Schaffer, T., Muller, S., Flogerzi, B., Seibold-Schmid, B., Schoepfer, A.M. \& Seibold, F. Anti-Saccharomyces cerevisiae mannan antibodies (ASCA) of Crohn's patients crossreact with mannan from other yeast strains, and murine ASCA IgM can be experimentally induced with Candida albicans. Inflamm. Bowel Dis. 13, 1339-1346 (2007).

31. Apostolopoulos, V., Pietersz, G.A., Gordon, S., Martinez-Pomares, L. \& McKenzie, I.F. Aldehyde-mannan antigen complexes target the MHC class I antigen-presentation pathway. Eur. J. Immunol. 30, 1714-1723 (2000).

32. Liu, L. et al. The beneficial effect of Rheum tanguticum polysaccharide on protecting against diarrhea, colonic inflammation and ulceration in rats with TNBS-induced colitis: the role of macrophage mannose receptor in inflammation and immune response. Int. Immunopharmacol. 8, 1481-1492 (2008).

33. Gazi, U. \& Martinez-Pomares, L. Influence of the mannose receptor in host immune responses. Immunobiology 214, 554-561 (2009). 
34. Wagner, H. Toll-like receptors in gastrointestinal diseases. Dig. Dis. 30 (Suppl 1), 74-77 (2012).

35. Cario, E. Therapeutic impact of toll-like receptors on inflammatory bowel diseases: a multiple-edged sword. Inflamm. Bowel Dis. 14, 411-421 (2008).

36. Fukata, M. et al. Toll-like receptor-4 is required for intestinal response to epithelial injury and limiting bacterial translocation in a murine model of acute colitis. Am. J. Physiol. Gastrointest. Liver Physiol. 288, G1055-G1065 (2005).

37. Rakoff-Nahoum, S., Paglino, J., Eslami-Varzaneh, F., Edberg, S. \& Medzhitov, R. Recognition of commensal microflora by Toll-like receptors is required for intestinal homeostasis. Cell 118, 229-241 (2004).

38. Ungaro, R. et al. A novel Toll-like receptor 4 antagonist antibody ameliorates inflammation but impairs mucosal healing in murine colitis. Am. J. Physiol. Gastrointest. Liver Physiol. 296, G1167-G1179 (2009).
39. Liu, Y. et al. TLR4 monoclonal antibody blockade suppresses dextransulfate-sodium-induced colitis in mice. J. Gastroenterol. Hepatol. 25, 209-214 (2010).

40. Fort, M.M. et al. A synthetic TLR4 antagonist has anti-inflammatory effects in two murine models of inflammatory bowel disease. J. Immunol. 174, 6416-6423 (2005).

41. Puimege, L. et al. Glucocorticoid-induced microRNA-511 protects against TNF by down-regulating TNFR1. EMBO Mol. Med. 7, 1004-1017 (2015).

42. Griffiths-Jones, S. The microRNA registry. Nucleic Acids Res. 32, D109D111 (2004).

43. Rice, P.J. et al. Oral delivery and gastrointestinal absorption of soluble glucans stimulate increased resistance to infectious challenge. J. Pharmacol. Exp. Ther. 314, 1079-1086 (2005).

44. De Palma, M. \& Naldini, L. Transduction of a gene expression cassette using advanced generation lentiviral vectors. Methods Enzymol. 346, 514529 (2002). 